\title{
FOSFATASA ALCALINA (ALP) Y RUNX2 EN CULTIVOS CELULARES DE OSTEOBLASTOS ESTIMULADOS CON CAMPO ELÉCTRICO
}

\author{
Jorge Arturo Rey Cubillos, MSc ${ }^{1}$, Profesor Asociado \\ Leonardo Lareo. PhD, Q.E.P.D ${ }^{2}$ PRofesor Titular \\ SANDRA GutiérRez, PhD. ${ }^{2}$ Profesora Asociada \\ MARCELA GODOY CORREDOR, BSc. ${ }^{1}$ JOVEN INVESTIGADOR \\ ${ }^{1}$ Universidad Militar Nueva Granada, Facultad de Medicina \\ ${ }^{2}$ Pontificia Universidad Javeriana
}

Recibido: Agosto 30 de 2012 Aceptado: Noviembre 15 de 2012

\section{Resumen}

\begin{abstract}
El objeto de este estudio fue identificar el estímulo eléctrico que debe aplicarse en cultivos celulares de osteoblastos (Ob) para aumentar la expresión del gen de Fosfatasa Alcalina (ALP) y el factor de transcripción Runx2. Se cultivaron Ob de la American Type Culture Collection (ATCC) Ref. CRL 11372. Los cultivos se estimularon del día cinco, cuando las células presentaron confluencia, hasta el día ocho. El estímulo aplicado a cada grupo experimental fue de $100 \mathrm{mV}, 200 \mathrm{mV}, 300 \mathrm{mV}, 400 \mathrm{mV}$ y $500 \mathrm{mV}$ respectivamente, se cultivó un grupo control no estimulado con cada grupo experimental. El campo eléctrico se generó con corriente alterna $(\mathrm{AC})$ y se aplicó mediante dos placas de aluminio ubicadas de forma lateral y paralela a los frascos de cultivo de $25 \mathrm{~cm}^{2}$. Los niveles de expresión de mRNA se midieron con la técnica quantitative reverse transcription polymerase chain reaction (qRT-PCR). Con la aplicación de campo generado con AC aumentó la expresión del factor de transcripción RunX2 en proporción directa al aumento del voltaje aplicado. La expresión del gen de ALP fue inversamente proporcional a la aplicación del estímulo y se identificó una diferencia significativa entre la presencia y ausencia del estímulo, siendo mayor en ausencia del estímulo. El campo eléctrico generó una señal que puede aumentar o disminuir la expresión de los genes que median la formación de tejido óseo. En el caso de Runx2, favoreció la diferenciación de células mesenquimales a $\mathrm{Ob}$ con la consecuente actividad de remodelación y formación del tejido óseo.
\end{abstract}

Palabras clave: Osteoblastos, fosfatasa alcalina, estímulo eléctrico, Runx2, expresión génica Abreviaciones: *FA, Fosfatasa Alcalina*; *Ob, Osteoblastos*

\section{ALKALINE PHOSPHATASE (ALP) AND RUNX2 IN CELL CULTURES STIMULATED OSTEOBLASTS ELECTRIC FIELD}

\begin{abstract}
The purpose of this study was to identify the electrical fields to be applied in osteoblast $(\mathrm{Ob})$ cell cultures, in order to increase the expression of Alkaline Phosphatase (ALP) gene and the transcription factor Runx2. Ob cultured where from the American Type Culture Collection (ATCC) Ref CRL 11372. Cell Cultures received stimulation at day five, when they showed a confluent monolayer organization until day eight. The stimulus applied to each experimental group was $100 \mathrm{mV}, 200 \mathrm{mV}, 300 \mathrm{mV}, 400 \mathrm{mV}$ and $500 \mathrm{mV}$ respectively, a control group was cultured without stimulation. The electric field is generated
\end{abstract}

* Correspondencia: Jorge Arturo Rey Cubillos, MSc. jorge.rey@unimilitar.edu.co, jrey@javeriana.edu.co Carrera 11 B No 135 A - 08. Interior 5 Apto 201. Barrio Lisboa. Tel. 571 - 6147298 
with altern current $(\mathrm{AC})$ and applied with two aluminum plates located parallel to $25 \mathrm{~cm}^{2}$ culture flasks. The mRNA expression levels were measured by reverse transcription quantitative technique polymerase chain reaction (qRT-PCR). Aplication of AC increased expression of transcription factor RunX2 in direct proportion to the applied voltage. The ALP gene expression was inversely proportional to the stimulus. Electric field can increase or decrease the expression of genes that mediate the formation of bone tissue. Favoring differentiation of mesenchymal cells to Ob.

Keywords: osteoblasts, alkaline phosphatase, electrical stimulation, Runx2, gene expression Abbreviations: *FA, alkaline phosphatase * * Ob, Osteoblasts *

\title{
FOSFATASE ALCALINA (ALP) E RUNX2 EM CULTIVOS CELULARES DE OSTEOBLASTOS ESTIMULADOS COM CAMPO ELÉTRICO
}

\begin{abstract}
Resumo
O objeto deste estudo foi identificar o estímulo elétrico que deve ser aplicado em cultivos celulares de osteoblastos (Ob) para aumentar a expressão do gene da Fosfatase Alcalina (ALP) e o fator de transcrição Runx2. Foram cultivados Ob da American Type Culture Collection (ATCC) Ref. CRL 11372. Os cultivos foram estimulados a partir do quinto dia, quando as células apresentaram confluência, até o oitavo dia. O estímulo aplicado a cada grupo experimental foi de $100 \mathrm{mV}, 200 \mathrm{mV}, 300 \mathrm{mV}$, $400 \mathrm{mV}$ e $500 \mathrm{mV}$ respectivamente, cultivou-se um grupo de controle não estimulado com cada grupo experimental. O campo elétrico foi gerado com corrente alternada (CA) e aplicou-se mediante duas placas de alumínio localizadas de forma lateral e paralela aos frascos de cultivo de $25 \mathrm{~cm}^{2}$. Os níveis de expressão de mRNA foram medidos com a técnica quantitative reverse transcription polymerase chain reaction (qRT-PCR). Com a aplicação do campo gerado com AC aumentou a expressão do fator de transcrição RunX2 em proporção direta ao aumento da voltagem aplicada. A expressão do gene de ALP foi inversamente proporcional à aplicação do estímulo e identificou-se uma diferença significativa entre a presença $e$ ausência do estímulo, sendo maior na ausência do estímulo. $O$ campo elétrico gerou um sinal que pode aumentar ou diminuir a expressão dos genes que mediam a formação de tecido ósseo. No caso de Runx2, favoreceu a diferenciação de células mesenquimais a $\mathrm{Ob} c 0 m$ a consequente atividade de remodelação e formação do tecido ósseo.
\end{abstract}

Palavras chave: Osteoblastos, fosfatase alcalina, estímulo elétrico, Runx2, expressão gênica Abreviações: *FA, Fosfatasa Alcalina*; *Ob, Osteoblastos*

\section{Introducción}

Uno de los grandes retos de las ciencias de la salud es el manejo del tejido óseo para su correcto desarrollo, regeneración y cicatrización. Se han desarrollado técnicas para reemplazos totales de estructuras óseas y técnicas con materiales de relleno para defectos menores. No existe consenso ante los resultados obtenidos con las diferentes técnicas: reemplazo, implantes, injertos y estímulos, para todas se reportan efectos positivos y negativos. La identificación de los campos eléctricos que activan de manera eficiente los procesos celulares y moleculares puede convertirse en una alternativa terapéutica para tratar los problemas que se presentan en huesos largos, planos e irregulares (1). Una de las técnicas más antiguas y de mayor difusión es conocida como "distracción osteogénica". En ella se realiza corticotomía preservando periostio a huesos largos y mediante tutores metálicos se traccionan los segmentos óseos para aumentar la longitud de la estructura inicial. En esta técnica el autor (Ilizarov) aplicó originalmente campos eléctricos y magnéticos a través de los tutores para favorecer la proliferación de osteoblastos y la mineralización de la matriz extracelular. Los campos alteran los receptores de membranas celulares $e$ incrementan la producción de factores de crecimiento, afectan el flujo de iones a través de la membrana y favorecen la formación de endotelio y capilares (2). La utilización de materiales exógenos para realizar injertos y 
rellenos, resulta en la creación de un soporte pasivo que no promueve actividad regenerativa $(3,4)$. Diferentes estudios clínicos, demuestran que el tejido óseo resultante de este tipo de terapias difiere en estructura y función del tejido óseo normal (5-7).

Las fallas en el proceso de regeneración y reparación del tejido óseo, se deben a la rápida proliferación de tejido conectivo fibroso. La ausencia de osteoblastos en la zona, explica la carencia de las moléculas necesarias para la formación y posterior mineralización de la matriz extracelular como son la fosfatasa alcalina $(\mathrm{ALP})$, osteonectina $(\mathrm{ON})$, osteopontina (OP), osteocalcina $(\mathrm{OC})$ también conocida como bone Gla protein (BGLA), sialoproteina ósea (BSP) y el factor de transcripción Runx2 (8-11).

Mutaz, B. y col. en su libro Bone grafts and bone substitutes, explican como en cultivos celulares se ha logrado experimentar con varios tipos de campo eléctrico para obtener diferentes resultados. Pezzet $F$ y col. demostraron que la aplicación de campos de baja intensidad y frecuencia sobre células en cultivo favorece la expresión de colágeno tipo I y II. Guerkov, H. y col. identificaron que la aplicación de campos modifica la expresión de factores de crecimiento y la actividad de ALP (11-13).

\section{Estimulación}

Las cargas mecánicas en el hueso, se convierten en señales eléctricas por mecanotransducción, lo cual genera un acoplamiento bioquímico y la respuesta de células efectoras (14).

Cuando una fuerza mecánica se aplica sobre el hueso, se separan las cargas positivas y negativas en el hueso y se registran dos polos con cargas opuestas que se orientan por la tensión de las moléculas que tienden a separarse. La diferencia neta de cargas en la superficie del hueso se conoce como "potencial eléctrico" $(15,16)$. La porción colágena de hueso posee un potencial generador de corrientes. Friedemberg, y col, demostraron que con la aplicación de corrientes exógenas por medio del uso de electrodos, se estimula el crecimiento y formación de células óseas $(17,18)$.

\section{Runx2}

En cuanto a las moléculas necesarias para la mineralización de la matriz extracelular, se define a Runt como un factor de transcripción en drosophila, el primero de varios factores de transcripción que se mantienen desde el C. Elegans hasta el Homo sapiens, el cual tiene afinidad con la subunidad $\alpha$ del Core binding factor alpha (Cbfa). En humanos existen tres genes relacionados que se denominan Core Binding Factor Alpha 1 (Cbfa1), Cbfa2 y Cbfa3; los cuales por medio de splicing alternativo, generan diferentes proteínas (19-21). El Cbfa1 es también conocido como Runx2, siendo éste el factor de transcripción especifico para osteogénesis (Runx2/AML3) y el determinante para la diferenciación de las células mesenquimales hacia linaje osteoblástico (22) bajo estímulo de genes específicos como OC, ALP, BSP, Colágeno tipo I (Col 1) y Colagenasa-3 (matriz de metaloproteinasa-13 [MMP-13]) (23).

\section{Fosfatasa Alcalina - ALP}

Se relaciona directamente con la mineralización de la matriz ósea; es un marcador de diferenciación del fenotipo osteoblástico y un indicador de formación y recambio óseo. La evidencia recolectada y publicada hasta la fecha permite establecer una relación directa entre la presencia de ALP y el normal desarrollo del tejido óseo en el cuerpo humano (24-27).

El estímulo eléctrico activa la ALP para formar un complejo fosfoprotéico que induce una mayor y mejor formación ósea en un lapso de 10 a 14 días in vivo y de 3 a 7 días in vitro (28).

\section{Materiales y métodos}

\section{Cultivos celulares}

Todas las pruebas se realizaron por triplicado. Se cultivaron $\mathrm{Ob}$ de la American Type Culture Collection (ATCC) Ref. CRL 11372. La densidad inicial fue de 1 x $10^{5}$ células por cada frasco de cultivo de $25 \mathrm{~cm}^{2}$. Se empleó el medio de cultivo Dulbecco `s Modified Eagle`s Medium/Nutrient Mixture F-12 HAM adicionado con $100 \mathrm{U} / \mathrm{ml}$ de penicilina, $100 \mu \mathrm{g} / \mathrm{ml}$ de estreptomicina, $0,5 \%$ de L-glutamina y $10 \%$ de suero fetal bovino. Los cultivos se mantuvieron con $5 \% \mathrm{CO}_{2}$ a $37^{\circ} \mathrm{C}$. Como células de control se utilizaron los mismos $\mathrm{Ob}$ de la ATCC sin la aplicación del estímulo eléctrico.

\section{Aplicación del estímulo}

El estímulo eléctrico se aplicó cuando las células alcanzaron su confluencia al quinto día. La aplicación se 
realizó durante cuatro (4) días. Recibieron estimulación con intensidad de campo de $100 \mathrm{mV}, 200 \mathrm{mV}, 300 \mathrm{mV}$, $400 \mathrm{mV}$ y $500 \mathrm{mV}$, respectivamente. Para cada grupo experimental se sembró un (1) grupo de control de las mismas células, sin la aplicación del estímulo.

\section{Estímulo}

El equipo de estimulación consta de una fuente de poder regulada que genera y asegura la estabilidad de los estímulos, se monitoreó la prueba con un electrómetro Keithley ${ }^{\circledR}$ como dispositivo lector y testigo del campo eléctrico. El área de estimulación eléctrica, se protegió con un apantallamiento de Faraday para evitar influencias de campos externos sobre los cultivos. El sistema completo se adaptó y se trabajó dentro de la incubadora. El sistema de estimulación tuvo que ser diseñado y fabricado y se patentó con el nombre de "Estimulador eléctrico para cultivos celulares basado en la aplicación de voltaje en un par de placas planas paralelas" cuyo numero de patente es No. 10-164983 y cuya Clasificación es IPC: A 61 N 7/00, H 02 B 1/00, otorgada por la Oficina de Patentes, Superintendencia de Industria y Comercio, Bogotá, Colombia.

Después del proceso de estimulación se registraron datos de morfología y viabilidad celular.

\section{Extracción de mRNA}

Para la obtención de mRNA de los Ob se empleó el reactivo TRIZOL LS Reagent de Invitrogen ${ }^{\circledR}$. Se agregó a la muestra $1 \mathrm{ml}$ de TRIZOL LS para la digestión de la misma. El mRNA se separó agregando cloroformo y se precipitó con alcohol isopropílico y etanol al $75 \%$; finalmente se resuspendió en agua DEPC y se almacenó a - $80^{\circ} \mathrm{C}$.

\section{Cuantificación de mRNA}

Se realizó mediante espectrofotometría utilizando el equipo TECAN (GENios ${ }^{\circledR}$ ) para determinar la absorbancia de los ácidos nucleicos a 260nm y calcular la cantidad de mRNA en nanogramos por microlitro $(\mathrm{ng} / \mu \mathrm{l})$.

\section{Verificación de integridad de mRNA}

La calidad del mRNA extraído se verificó en geles de agarosa al $2 \%$ con formaldehído al $37 \%$. Los geles se tiñeron con bromuro de etidio y se corrieron en Buffer
MOPS $1 \mathrm{X}$ durante $60 \mathrm{~min}$ a $100 \mathrm{~V}$ para la posterior visualización de bandas características de RNA (28S y $18 S$ ) al ser expuestos a luz ultravioleta (UV). Se sembraron $3 \mathrm{ml}$ de cada muestra junto con formamida, Buffer MOPS 10X y Buffer de carga (Fig. 1).

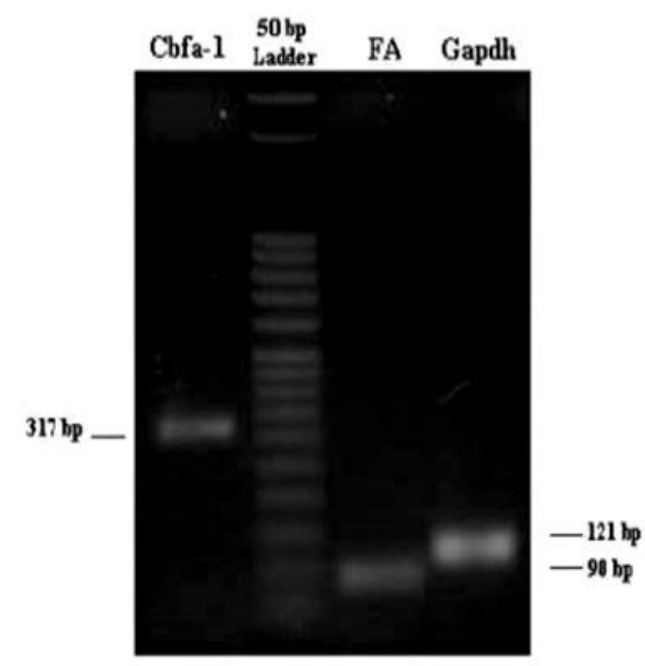

Figura. 1 Verificación de integridad del RNAm extraido

Síntesis de cDNA.

Se tomó $1 \mu \mathrm{g}$ de mRNA del obtenido de los Ob cultivados, este se sometió a transcripción reversa utilizando el Kit SuperScript ${ }^{\circledR}$ First Strand - Synthesis System de Invitrogen ${ }^{\circledR}$, para obtener el DNA complementario (cDNA).

Reacción en cadena de la polimerasa con transcriptasa inversa - método cuantitativo (QRT-PCR)

El cDNA sintetizado en la transcripción reversa se utilizó para cuantificar la expresión de los genes que codifican para ALP y Runx2. El procedimiento se llevó a cabo en un equipo Opticon II de MJ Research ${ }^{\circledR}$ usando el fluorocromo SyberGreen ${ }^{\circledR}$. La calidad del cDNA se controló realizando el mismo procedimiento para el gen GAPDH, el cual se utilizó como control interno o "housekeeping". El cDNA obtenido se amplificó mediante qRT-PCR utilizando los primers específicos para cada gen. Los primers para ALP y Runx2 fueron tomados de la literatura $(17,28)$ y estandarizados. Los primers para GAPDH fueron diseñados con el programa OLIGO6 siguiendo las recomendaciones de Bustin, SA (Tabla1) (29). Adicionalmente los productos amplificados se insertaron en vectores plasmídicos (Corpogen Vector TOPO 2.1) para generar curvas standard y realizar el 
Tabla 1. Secuencia de los primers utilizados

\begin{tabular}{|l|l|l|l|}
\hline \multicolumn{1}{|c|}{ Primer } & \multicolumn{1}{|c|}{ Secuencia } & \multicolumn{1}{c|}{$\begin{array}{c}\text { Tamaño } \\
\text { de banda }\end{array}$} & \multicolumn{1}{c|}{ Publicado por } \\
\hline Runx2-F & 3' ACTGGGCCCTTTTTCAGA 5' $^{\prime}$ & $317 \mathrm{pb}$ & Huang L (2004) \\
\hline Runx2-R & 3' GCGGAAGCATTCTGGAA 5' $^{\prime}$ & & \\
\hline ALP-F & 5'CGTGGCTAAGAATGTCATCATGTT 3'x $^{\prime}$ ' $89 \mathrm{pb}$ & Haase HR (2003) \\
\hline ALP-R & 5' TGGTGGAGCTGACCCTTGA 3' $^{\prime}$ & & \\
\hline GAPDH-F & 5'GGAGTCAACGGATTTGGT 3' $^{\prime}$ & $121 \mathrm{pb}$ & Huang L (2004) \\
\hline GAPDH-R & 5' GTGATGGGATTTCCATTGAT 3' & & \\
\hline
\end{tabular}

análisis cuantitativo de la expresión de cada uno de los genes en estudio.

\section{Resultados}

\section{Expresión de RUNX2 y alp}

Después de 4 días de la aplicación del estímulo con $100 \mathrm{mV}, 200$ mV, 300 mV, 400 mV y 500 mV, se observó que el factor de transcripción Runx2, se incrementó progresivamente en proporción al incremento del estímulo. Los datos del grupo control para Runx2, fueron 113,734 moléculas por microlitro $(\mathrm{mol} / \mu \mathrm{L})$. Este factor inició su incremento progresivo de expresión con estímulo de $100 \mathrm{mV}$ en $227,013 \mathrm{~mol} / \mu \mathrm{L}$ y aumentó su expresión hasta $2799,415 \mathrm{~mol} / \mu \mathrm{L}$ con el estímulo más alto de $500 \mathrm{mV}$, esta fue la mayor expresión del factor para toda la prueba, en contraste el gen de Fosfatasa Alcalina (ALP) decreció de manera inversamente proporcional con el incremento del estímulo. La expresión de ALP en el grupo control fue de $523,117 \mathrm{~mol} / \mu \mathrm{L}$ y en contraste se observó una expresión de $61,415 \mathrm{~mol} /$ $\mu \mathrm{L}$ con el estímulo de $100 \mathrm{mV}$ hasta $2,118 \mathrm{~mol} / \mu \mathrm{L}$ con el estímulo de intensidad de $500 \mathrm{mV}$ (Figura 2).

El análisis realizado mediante T-Student (Tabla 2) permitió evidenciar la eficiencia del estímulo eléctrico para incrementar la expresión del factor de transcripción Runx2. Con el estímulo de $300 \mathrm{mV}$ observamos el dato de mayor diferencia entre el dato de control y la expresión en todas las demás intensidades, lo anterior se explica porque en $300 \mathrm{mV}$ se observa mayor varianza que en las otras intensidades, sin embargo, esto no afecta el aumento progresivo identificado en $\mathrm{mol} / \mu \mathrm{L}$ lo que permite concluir que el aumento de la expresión es proporcionalmente progresivo y tiene relación directa con el incremento de las intensidades de menor a mayor valor.

\section{Discusión}

Los reportes de la literatura científica corroboran que la aplicación de campos eléctricos de baja frecuencia y energía favorecen los procesos de proliferación y diferenciación celular y por lo mismo repercuten favorablemente en el metabolismo y la regeneración de tejido óseo (16), en este estudio se encontró que en efecto la presencia del campo favorece la expresión de Runx2, factor de transcripción temprano indispensable para que las células mesenquimales de médula ósea se diferencien al linaje osteoblástico (30-33). McLeod

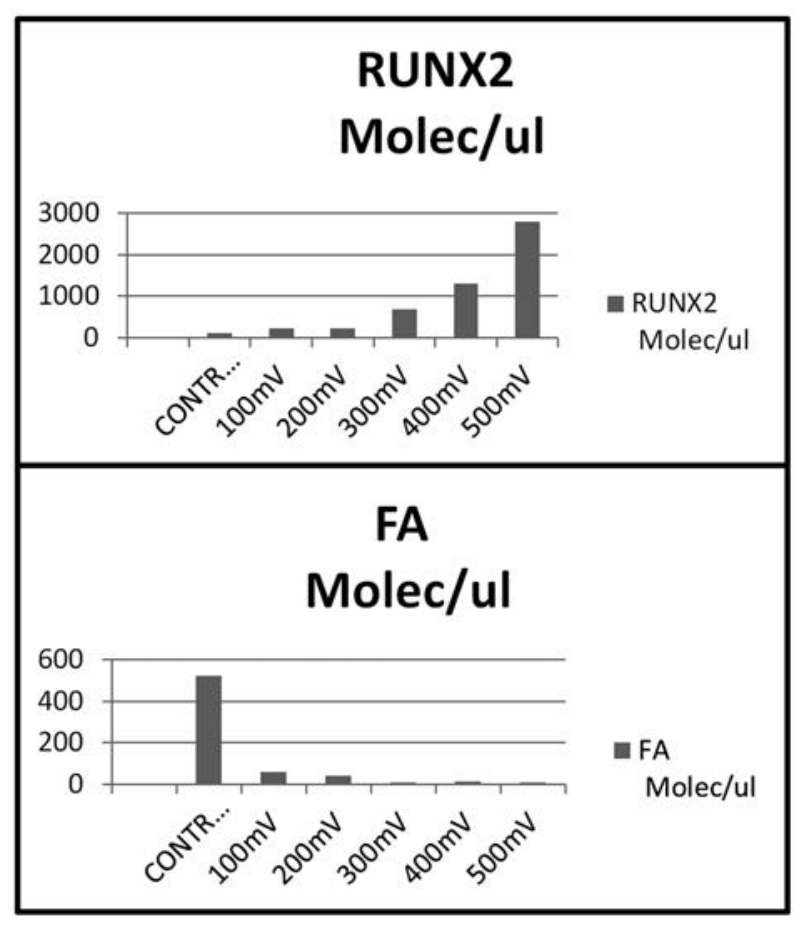

Figura. 2 Expresión relativa del factor de transcripción $R U N X 2$ y el gen ALP 
y Brighton (34) respectivamente encontraron que los estímulos eléctricos juegan un importante papel en el metabolismo óseo, aunque este mecanismo no se haya elucidado completamente. Con este estudio se está ayudando a clarificar algunos de los eventos moleculares que suceden en este proceso, para soportar estudios posteriores.

Los resultados obtenidos al someter los cultivos celulares de $\mathrm{Ob}$ a estímulos eléctricos con diferentes voltajes, favoreció la actividad celular expresando marcadores con fenotipo osteoblástico: factor de transcripción Runx2 y expresión del gen ALP. Cbfa1 o Runx2, se constituye como uno de los reguladores más potentes de la diferenciación osteoblástica y ALP es una proteína de superficie que podría participar en la regulación de la proliferación, migración y diferenciación de las células osteoblásticas además de la mineralización de la matriz extracelular del hueso.

La aplicación posterior de estos y otros resultados a nivel clínico podría coadyuvar a la regeneración partes de estructuras óseas en un individuo que haya sufrido algún tipo de trauma óseo.

El descenso en la expresión del gen de ALP coincide con datos reportados por Basso y Ozawa $(34,35)$. Estos resultados podrían estar explicando que la activación de los procesos de diferenciación y proliferación celular requiere de la inactivación o disminución del proceso de mineralización de matriz extracelular, mediado por ALP.

La proyección de aplicación de estos resultados puede cobrar relevancia clínica en el desarrollo de técnicas, que permitan regenerar tejido sin la colocación de injertos o materiales de relleno. La importancia de ésto, radica en que actualmente es muy elevado el número de pacientes que debe someterse a procesos quirúrgicos para rellenar e injertar materiales en defectos óseos, los cuales a pesar de ser exitosos aun se encuentran dentro de la clasificación de procesos de cicatrización y reparación del tejido, mas no dentro del área de la regeneración ósea.

\section{Agradecimientos}

Agradecimiento a las universidades en las cuales se logró la integración de esfuerzos para el desarrollo de todas las etapas experimentales del proyecto. Universidad Militar Nueva Granada, Pontificia Universidad Javeriana, Universidad Nacional de Colombia, Instituto Stem, Medicina Regenerativa.

\section{Conflictos de interés}

Los autores expresan que no presentan ningún conflicto de interés en la publicación de los resultados de esta investigación.

\section{Referencias}

1. Hronik-Tupaj M, Rice WL, Cronin-Golomb M, Kaplan DL, Georgakoudi I. Osteoblastic differentiation and stress response on human mesenchymal stem cells exposed to alternating current electric fields. Engineering OnLine. 2011; 10:9

2. Anglen J. Enhancement of fracture healing with bone stimulators. Techniques In Orthopaedics. 17(4): 506-514. 2003.

3. Buser D. Guided bone regeneration in implant dentistry. Hong Kong: Editorial Quintessence; 1994. p. 31-49.

4. Mutaz B, Habal A, Hari R. Bone grafts and bone substitutes. $1^{\mathrm{a}}$ ed. Saunders; 1992

5. Byl NN, McKenzie AL, West JM, Whitney JD, Hunt TK, et al. Pulsed microamperage stimulation: a controled study of healing of surgically induced wounds in yucatan pigs. Phys. Ther. 1994; 74(3): 201-18.

6. Ciombor DM, Aaron RK. Influence of electromagnetic fields on endochondral bone formation. J. Cell-Biochem. 1993; 52(1): 37-41.

7. Buser D. Guided bone regeneration in implant dentistry. Hong Kong: Editorial Quintessence; 1994. p. 13-30.

Tabla 2. Análisis de varianza por T-Student

\begin{tabular}{|c|c|c|c|c|c|c|c|c|}
\hline \multirow{9}{*}{ 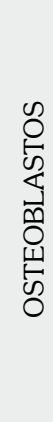 } & \multicolumn{4}{|c|}{ CBFA1 } & \multicolumn{4}{|c|}{ FA } \\
\hline & \multirow[t]{2}{*}{ Varianza } & \multicolumn{3}{|c|}{ DATOS } & \multirow[t]{2}{*}{ Varianza } & \multicolumn{3}{|c|}{ DATOS } \\
\hline & & 1 & 2 & 3 & & $\mathbf{1}$ & 2 & 3 \\
\hline & $500 \mathrm{mV}$ & 16414,5572 & 1904,01323 & 10121,366 & 1,0378255 & 1,104601 & 0,966289 & 0,004761 \\
\hline & $400 \mathrm{mV}$ & 8722,54293 & 8991,97028 & 8,608356 & 0,9804375 & 1,09025 & 0,013225 & 0,855625 \\
\hline & $300 \mathrm{mV}$ & 1,0717 & 0,6889 & 1,3456 & 1,314469 & 1,311025 & 9E-06 & 1,317904 \\
\hline & $200 \mathrm{mV}$ & 1,6553855 & 0,012321 & 1,792921 & 0,271548 & 0,242064 & 0,002916 & 0,298116 \\
\hline & $100 \mathrm{mV}$ & 0,9812045 & 0,994009 & 0,968256 & 0,711124 & 0,6241 & 0,009604 & 0,788544 \\
\hline & $\operatorname{Sin} E$ & 2,112545 & 2,480625 & 1,661521 & 0,017701 & 0,000016 & 0,018225 & 0,017161 \\
\hline
\end{tabular}


8. Castro D. Estudio comparativo de materiales de relleno óseo, análisis histológico, clínico y radiológico: Bogotá:T.G; 1994. p. 14-97.

9. Dogan E. Monotoring hormone replacement therapy by biochemical markers of bone metabolism in menopausal women, Postgraduate Medical Journal. 78(926): 727-738.

10. Duplomb L. Embryonic stem cells: new tool to study osteoblasts and osteoclast differentiation. Stem Cells Published On Line. Acceso 9 de noviembre de 2006; Disponible en: 10.1634/ Stemcells.2006-0395. (www.Stemcells.Com)

11. Nieden Z. In vitro differentiation of embryonic stem cells into mineralized osteoblasts. Differentiation. 2003; 71(1): 18-27.

12. Pezzet F. Efects of pulsed electromagnetic fields on human chondrocytes. J. Calcified Tissue International. 1999; 65(5): 396-401

13. Guerkov HH, Lohmann CH, Liu Y, Dean DD, Simon BJ, Heckman JD, et al. Pulsed elctromagnetic fields increase growth factor release by nonunion cells. Clin Orthop Relat Res. 2001; 1(384): 265-79.

14. Schriefer JL, Warden SJ, Saxon LK, Robling AG, Turner CH. Cellular accommodation and the response of bone to mechanical loading. J Biomech. 2005; 38(9): 1838-45.

15. Shamos Mh. Piezoelectric effect in bone. Nature. 1963; 197:81.

16. Fukada E, Yasunda I. The piezo-electric effect of bone. J. Physiol. Soc. Japan. 1957; 12: 1158-1162.

17. Friedenberg, Z, Brighton CT. Bioelectric Potentials in Bone. J. Bone Joint S Urg., 1.966. 48:915-923.20.

18. Tsai M, Li W, Tuan RS, Chang WS. Modulation of osteogenesis in human mesenchymal stem cells by specific pulsed electromagnetic field stimulation. J Orthop Res. 2009; 27(9): 1169-1174.

19. Karsenty G, Ducy P, Starbuck M, Priemel M, Shen J, Geoffroy $\mathrm{V}$, et al. $\mathrm{Cbfa} 1$ as a regulator of osteoblast differentiation and function. Bone. 1999; 25(1): 107-108.

20. Ducy P. Cbfa1: a molecular switch in osteoblast biology. Developmental Dynamics. 2000; 219: 461-471.

21. Ziros PG, Basdra EK, Papavassiliou AG. Runx2: Of Bone And Stretch. Int. J. Biochem. Cell Biol. 2008; 40: 1659-1663

22. Pierre JM. Review transcription factors controlling osteoblastogenesis. Archives of Biochemistry and Biophysics. 2008; 473; 98-105

23. Ducy P, Zhang R, Geoffroy V, Ridall AL, Karsenty G. Osf2/cbfa1: a transcriptional activator of osteoblast differentiation. Cell 89, 1997; 89(5): 747-754.
24. Fishman WH, Ghosh NK. Isoenzymes of human alkaline phosphatase. Adv Clin Chem. 1967; 10: 255-370

25. Fallon MD, Whyte MP, Teitelbaum SL. Stereoespecific inhibition of alkaline phosphatase by l- tetramisole prevent in vitro cartilage calcification. Lab Invest. 1980; 43(6): 489-494.

26. Mitchell J. Structure of the human liver/bone/kidney/ alkaline phosphatase gene. J. Biol Chem. 1988; 263(24):12002-12010.

27. Jansen J, Van der Jagt O, Punt BJ, Verhaar J, Van Leeuwen J, Weinans $\mathrm{H}$, et al. Stimulation of osteogenic differentiation in human osteoprogenitor cells by pulsed electromagnetic fields: an in vitro study. BMC Musculoskeletal Disorders. 2010; 11:188.

28. Huang L, Teng X.Y, Cheng Y.Y, Lee K.M, Kumta S.M. Expression of preosteoblast markers and Cbfa- 1 and Osterix gene transcripts in stromal tumour cells of giant cell tumour of bone. J. Bone. 2004; 34: 393-401.

29. Haase HR, Ivanovski S, Waters MJ, Bartold PM. Growth hormone regulates osteogenic marker mRNAexpression in human periodontal fibroblasts and alveolar bone-derived cells. J Periodont Res. 2003; 38: 366-374.

30. Bustin, SA. Quantification of mRNA using real-time reverse transcription PCR (RT-PCR): trends and problems. Journal of Molecular Endocrinology. 2002; 29: 23-39.

31. K.H. Lau, S. Kapur, C. Kesavan, D.J. Baylink. Up-regulation of the WNT, estrogen receptor, insulin-like growth factor-I, and bone morphogenetic protein pathways in C57BL/6J osteoblast as opposed to $\mathrm{C} 3 \mathrm{H} / \mathrm{HeJ}$ osteoblast in part contributes to the differential anabolic response to fluid shear. J. Biol. Chem. 2006; 281(14): 9576-88.

32. McLeod KJ, Rubin CT. Frequency specific modulation of bone adaptation by induced electric fields. J Theor Biol. 1990; 145(3): 385-96.

33. Brighton CT, Okereke E, Pollack SR, Clark CC. In vitro bone-cell response to a capacitively coupled electrical field. The role of field strength, pulse pattern, and duty cycle. Clin Orthop Relat Res. 1992; (285): 255-62.

34. Basso N, Heersche JN. Characteristics of in vitro osteoblastic cell loading models. Bone. 2002; 30(2): 347-51.

35. Ozawa H, Imamura K, Abe E, Takahashi N, Hiraide T, Shibasaki Y, et al. Effect of a continuously applied compressive pressure on mouse osteoblast-like cells (MC3T3-E1) in vitro. J Cell Physiol. 1990; 142(1): 177-85. 